\title{
IDENTIFICAÇÃO DE GÊNERO E CONTORNO CORPORAL PARA ESTIMATIVA DE SOMATOTIPO
}

\author{
Gentil Auer Neto*, Patrick Marques Ciarelli*, Evandro Ottoni Teatini Salles* \\ *Universidade Federal do Espírito Santo, Vitória, Espírito Santo, Brasil
}

Emails: auergentil@hotmail.com,patrick.ciarelli@ufes.br,evandro.salles@ufes.br

\begin{abstract}
In the areas of health and physical education, some approaches with patients and athletes are defined according to the somatotype of individuals, whose automatic classification based on images of the body has been researched with a focus on reducing the complexity of the exams. One of the difficulties of this approach is the requirement of lighting control and a homogeneous background distinct from the human body. The present work consists in applying the Mask R-CNN technique to images of people in uncontrolled environments to obtain two important information for estimating the somatotype: the gender and the body contour at a pixel level. To evaluate the performance of the method, images of general people and bodybuilders were tested. The classification reached $95.4 \%$ accuracy for males and $87.7 \%$ for females. Body contours were segmented correctly even in uncontrolled environment images.
\end{abstract}

Resumo: Nas áreas da saúde e educação física, algumas abordagens com pacientes e atletas são definidas de acordo com o somatotipo dos indivíduos, cuja classificação automática baseada em imagens do corpo tem sido pesquisada com foco em diminuir a complexidade dos exames. Uma das dificuldades dessa abordagem é a necessidade de controle de iluminação e de um fundo homogêneo distinto do corpo humano. O presente trabalho consiste em aplicar a técnica Mask R-CNN em imagens de pessoas em ambientes não controlados para obter duas informações importantes para a estimativa do somatotipo: o gênero do indivíduo e o contorno corporal em nível de pixel. Para avaliar o desempenho do método, foram testadas imagens de população geral e de fisiculturistas. A classificação atingiu 95,4\% de acurácia para o gênero masculino e $87,7 \%$ para o feminino. Os contornos corporais foram segmentados corretamente mesmo em imagens em ambientes não controlados.

Keywords: Somatotype; Segmentation; Classification; Convolutional Neural Networks; Mask R-CNN

Palavras-chaves: Somatotipo; Segmentação; Classificação; Redes Neurais Convolucionais; Mask R$\mathrm{CNN}$

\section{INTRODUÇÃO}

Detecção e segmentação de pessoas em imagens são temas bastante discutidos na área de visão computacional. Sistemas de vigilância e controle de acesso, sistemas de apoio ao motorista, aplicações médicas, inspeções industriais e localização de pessoas são algumas das áreas exploradas.

Somatotipo é o termo utilizado para representar a estrutura física do corpo humano, sendo formado pela combinação de três folhetos embrionários: endomorfismo, mesomorfismo e ectomorfismo (Sheldon et al., 1940). Na área de educação física e saúde, a identificação do somatotipo de cada indivíduo é uma métrica relevante na análise de pacientes e atletas, sendo possível observar o efeito de determinadas doenças e características de nutrição de crianças no corpo humano através do somatotipo, bem como auxiliar na orientação de treinamentos (Da Silva et al., 2003). Na prática, também é possível identificar por exemplo o esporte mais indicado para cada indivíduo visando benefícios para a saúde, ou ainda predizer o potencial de crianças para determinadas modalidades que possuam características corporais bem definidas em seus atletas (Gonçalves, 2017) e (Jaksic e Cvetković, 2009).

O método clássico para obter o somatotipo utiliza aparelhos antropométricos, como balanças, adipômetros e fitas, que nem sempre são portáteis ou facilmente acessíveis, além de tomar um considerável tempo para a obtenção das medidas e requisitar profissionais treinados. Em Gonçalves (2017) foi proposto um método para a identificação automática de somatotipo por imagens, resultando em medidas muito próximas aos valores obtidos pelo método clássico, com as vantagens de ser muito mais rápido e não necessitar de profissionais experientes. Entretanto, algumas das limitações do método proposto são que as imagens deveriam ser obtidas em ambientes controlados e previamente preparados, com iluminação uniforme, fundo homogêneo e distinto do corpo 
humano, uma pessoa por imagem, etc., além de ser fornecido por meio externo o peso e o gênero do indivíduo.

Falhas de iluminação, presença de ruídos, sobreposição de objetos, falta de padronização de posicionamento, além de grande variabilidade nos cenários e planos de fundo são barreiras que vêm sendo vencidas, possibilitando a extração de características e construção de conhecimento baseado em imagens obtidas nesses ambientes desafiadores (Parolin, 2011).

Algumas técnicas de aprendizado de máquina possibilitam detectar objetos, como faces, e rapidamente descartar fundos de imagens (Viola et al., 2004), ou ainda estimar características dimensionais de objetos em imagens abordando conceitos de profundidade monocular e modelos probabilísticos (Saxena et al., 2005).

Inovações recentes têm proporcionado soluções cada vez mais robustas, motivadas pela grande variedade de aplicações baseadas em CNN - Convolutional Neural Networks (Fukushima, 1988 e LeCun et al., 1989). Um exemplo são as redes neurais profundas, que são compostas por várias camadas de processamento não linear, onde a saída de cada camada anterior alimenta a entrada de sua camada posterior (Deng, 2014).

Nos últimos anos, avanços nas pesquisas utilizando redes profundas, por exemplo, com os algoritmos R-CNN - Region with CNN features (Girshick et al., 2014), FCN - Fully Convolutional Networks (Long et al., 2015), Fast R-CNN Fast Region-based Convolutional Network (Girshick et al., 2015), Faster R-CNN (Ren et al., 2015), FCIS - Fully Convolutional Instance-aware Semantic Segmentation (Li et al., 2017) e Mask R-CNN - Mask Region-based Convolutional Network (He et al., 2018) possibilitaram resultados promissores na segmentação de pessoas e classificação de objetos em ambientes não controlados, aliando ainda avanços relevantes em aspectos de custo computacional e acurácia.

Este trabalho visa dar continuidade à metodologia proposta por Gonçalves (2017), de forma a reduzir as suas limitações e facilitar a automatização da estimativa do somatotipo. Sendo assim, o objetivo deste trabalho é aplicar técnicas de redes neurais profundas em imagens de pessoas em ambientes não controlados, segmentar os contornos corporais e estimar o gênero. Para a segmentação apropriada dos indivíduos é proposto usar a rede neural profunda Mask R-CNN, pois ela realiza a segmentação em nível de pixel, sendo bastante precisa a sua segmentação, mesmo em condições adversas. Torna-se um estudo que, aliado ao proposto em (Gonçalves, 2017), avançará no sentido de complementar uma técnica robusta e automática de obtenção do somatotipo, ampliando possibilidades de ambientes para captura de imagens para realização de exames de pacientes e atletas, inclusive possibilitando avaliar em uma única imagem mais de um indivíduo, continuando o trabalho desenvolvido por Gonçalves (2016 e 2017).

A estrutura deste trabalho é tal que na Seção 2 é apresentado o embasamento teórico, na Seção 3 é descrita a técnica abordada, na Seção 4 é detalhada a metodologia de realização deste trabalho, na Seção 5 são apresentados os resultados e na Seção 6 é feita a conclusão e apresentados trabalhos futuros.

\section{EMBASAMENTO TEÓRICO}

Existem técnicas que utilizam redes neurais convolucionais para determinar se uma imagem contém um determinado objeto. Um exemplo é a AlexNet, uma arquitetura contendo cinco camadas de convolução, algumas delas seguidas por camadas de max-pooling e três camadas do tipo totalmente conectadas (Krizhevsky et al, 2012). Foi a arquitetura vencedora do ILSVRC 2012 (ImageNet Large-Scale Visual Recognition Challenge), e a responsável por popularizar as redes neurais convolucionais (CNN).

Outras arquiteturas mais avançadas indicam onde o objeto se encontra na imagem, realizando buscas por regiões de interesse, algo que foi introduzido pela R-CNN (Girshick et al. 2013), que funciona com três etapas: primeiro é feita a proposição de regiões candidatas, depois a extração de características de cada região utilizando uma CNN e finalmente é realizada a classificação de cada região utilizando support vector machine (SVM). A segunda etapa pode funcionar com diferentes arquiteturas de redes convolucionais, dentre elas a já citada AlexNet. Um problema dessa abordagem é o custo computacional, uma vez que para cada imagem de entrada são geradas aproximadamente duas mil regiões de interesse a serem processadas e classificadas.

Anos depois foi desenvolvida a Faster R-CNN (Ren et al., 2015), que para reduzir o custo computacional substituiu a busca seletiva por regiões de interesse por um modelo de rede convolucional pré-treinado que atua diretamente na imagem de entrada e gera um mapa de características, sendo posteriormente aplicado a uma segunda rede denominada Region Proposal Network (RPN), que funciona como uma janela deslizante que analisa o mapa de características, realiza supressão de não-máximos e evita o excesso de regiões candidatas por meio da técnica de pooling da região de interesse (RoI). A Figura 1 mostra um diagrama esquemático do RoI pooling, que funciona redimensionando as imagens para um tamanho fixo, depois obtém um novo vetor de características para cada RoI, prediz a categoria do objeto e o associa à sua localização na imagem original.

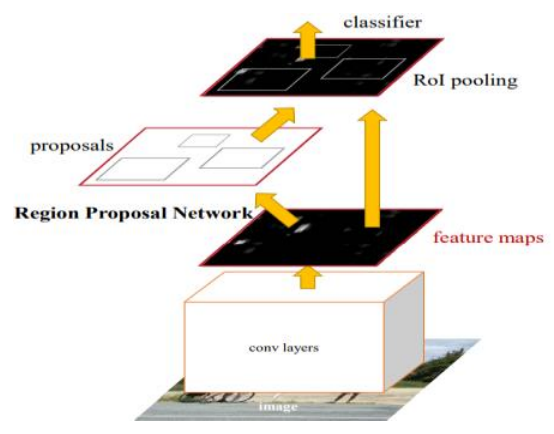

Fig. 1 Region Proposal Network e RoI pooling (Ren et al., 2015) 
Indo além, recentemente foi desenvolvido um algoritmo capaz de detectar e segmentar objetos em nível de pixels chamado Mask R-CNN (He et al., 2018), capaz de realizar tal tarefa para múltiplas instâncias de objetos em uma imagem, inclusive quando há sobreposição entre eles. Em outras palavras, ele levou a Faster R-CNN para o nível de pixel. Uma descrição mais detalhada dela é apresentada na Seção 3.

\section{TÉCNICA ABORDADA}

Dada uma imagem de entrada, a Mask R-CNN tem como saídas as classificações dos objetos identificados nela, seus bounding boxes e suas máscaras, que são exatamente a aproximação mais fiel possível encontrada para representar o contorno do objeto, isto é, em nível de pixel. Ela é uma extensão da Faster R-CNN e adiciona uma ramificação responsável por realizar a segmentação de instâncias. Funcionando em duas etapas principais, primeiramente ela encontra regiões candidatas de maneira semelhante à Faster R-CNN, implementando uma RPN. No segundo estágio é que entra a nova ramificação citada, responsável por paralelamente predizer e classificar a máscara do objeto.

Mask R-CNN realiza o alinhamento pixel a pixel da máscara utilizando uma técnica criada por seus autores denominada RoI Align. Ela atua sobre cada RoI do mapa de características obtido da imagem original e o divide em partes menores, depois seleciona um determinado número de pixels da fronteira de cada parte, dependendo do tamanho da máscara usada, e calcula um novo valor para cada parte do mapa por meio de interpolação bilinear, prevalecendo no final o mais representativo deles em cada uma dessas regiões. A Figura 2 exemplifica um caso de RoI Align para um mapa de características de tamanho $5 \times 5$, em que quatro máscaras de tamanho $2 \times 2$ são aplicadas e, após a interpolação bilinear seguida de supressão de não máximos ou cálculo de média, resulta em uma representação $2 \times 2$ para a RoI. O tamanho da máscara do RoI Align é um hiperparâmetro fixo definido antes do treinamento.

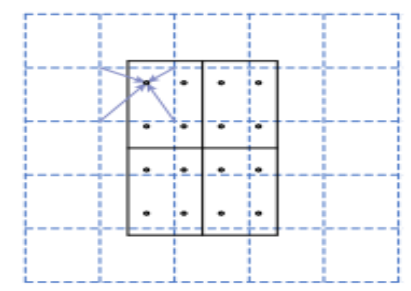

Fig. 2 RoI Align (He et al., 2018)

Durante o treinamento, para cada RoI candidata é definida uma máscara de dimensão $\mathrm{m}$ x m utilizando uma rede FCN (Long et al., 2015). Uma RoI proposta é considerada positiva se a interseção dela com a região do objeto da imagem original for de pelo menos $50 \%$, e quando isto ocorre é definida como uma máscara alvo. Uma máscara alvo é avaliada para todas as classes de treinamento, e por fim é definida uma probabilidade de ela estar associada a cada classe, prevalecendo a que possuir o maior valor.

A Figura 3 mostra a arquitetura resumida da técnica Mask RCNN, com a seleção de regiões de interesse seguida do alinhamento e finalizando com a segmentação e classificação de cada instância encontrada na imagem.

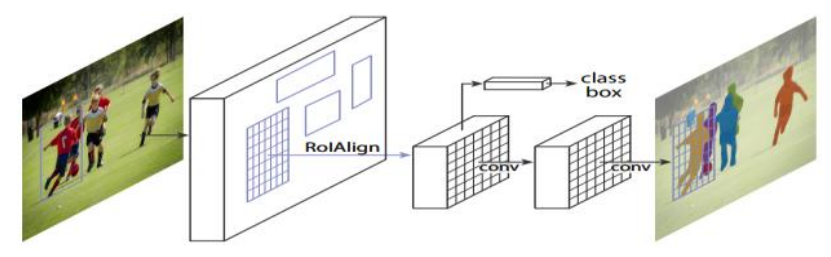

Fig. 3 Arquitetura Mask R-CNN (He et al., 2018)

Foram escolhidos dois backbones para a realização dos testes desse trabalho com a Mask R-CNN, sendo eles ResNet50 e ResNet101 (He et al., 2016). Com a ResNet foi introduzido o conceito de conexões de salto, responsáveis por atenuar o problema de desaparecimento do gradiente, que ocorre quando em redes com muitas camadas o valor do gradiente propagado é muito pequeno. Além disso, nela foi implementada uma função que garante que uma camada superior tenha um desempenho minimamente igual ao de uma camada inferior, e nunca pior.

\section{METODOLOGIA}

\subsection{Bases de dados}

Para a realização do trabalho foram utilizadas duas fontes para montar a base de dados, sendo a primeira delas a base WIKI (Rothe et al., 2016), que disponibiliza imagens de forma gratuita na internet. Dela foram escolhidas 200 imagens de homens e 200 imagens de mulheres. A base WIKI pode ser acessada em https://data.vision.ee.ethz.ch/cvl/rrothe/imdb-wiki/.

A segunda fonte para a base de dados é a mesma utilizada por (Gonçalves, 2017), contendo imagens de atletas fisiculturistas, sendo 72 homens e 46 mulheres. A validação realizada nessa segunda base é a referência para trabalhos sequentes, rumo à automatização da estimativa do somatotipo.

Para a classificação de gênero foram definidos os rótulos masculino e feminino e geradas as máscaras de treinamento utilizando a ferramenta online LabelMe (Russel et al., 2008) nas imagens da base WIKI e também nas imagens dos fisiculturistas. O conjunto de dados de treinamento consiste, portanto, de imagens originais e dos bouding boxes gerados com o uso do LabelMe sob os objetos de interesse. A Figura 4 exibe duas imagens de treinamento.
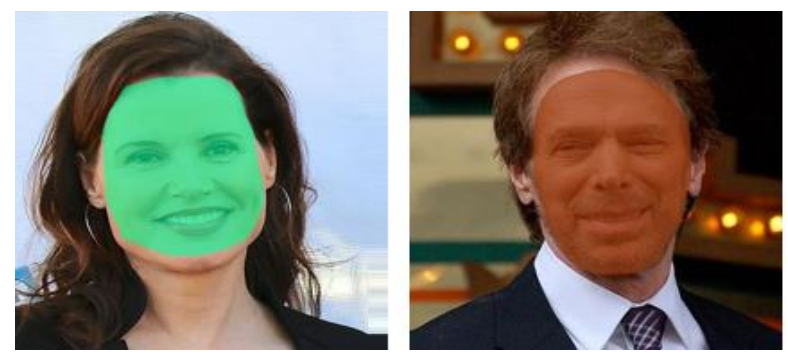

Fig. 4 Imagens do conjunto de treinamento com máscaras para classificação de gênero baseada em face 
Além disso, após alguns testes iniciais, foram inseridas ao grupo de treinamento 100 imagens da base WIKI sem nenhum indivíduo, portanto também sem rótulo, visando melhorar a acurácia do algoritmo, totalizando 618 imagens. Do total utilizado, $60 \%$ da base de dados foi separada para treinamento, e o restante foi utilizado para teste.

\subsection{Estratégia}

A estratégia adotada nesse trabalho consiste em duas etapas: primeiro é feita a identificação e segmentação do corpo completo do indivíduo, e depois é feita a classificação do gênero analisando apenas a face. $\mathrm{O}$ objetivo é fornecer como saída do sistema duas informações: se o indivíduo é do gênero masculino ou feminino, além da imagem segmentada com o contorno o mais próximo possível do corpo real da pessoa, permitindo que esses dados sejam utilizados em um sistema posterior de estimativa do somatotipo.

Para essa tarefa foram utilizados como backbones da Mask R-CNN dois modelos ResNet. Para a detecção e segmentação de pessoas foi aplicado primeiramente um modelo ResNet50 e posteriormente um modelo ResNet101, ambos contendo pesos pré-treinados para segmentação de pessoas tendo sido treinados na base Common Objects in Context (COCO) (Lin et al., 2014).

Para a classificação de gênero foi aproveitado o modelo ResNet50, porém nessa etapa foi realizado o retreinamento da rede em quatro abordagens distintas.

Primeiro foi feito o treinamento completo do modelo e não foram utilizadas imagens de classe negativa, isto é, sem indivíduos nas fotos. Esse método é apresentado como sem transfer learning; sem classe negativa na Seção 5.

No segundo momento, foi inserida ao treinamento a classe negativa, o que aumentou ligeiramente a acurácia do algoritmo, diminuindo a ocorrência de falsos positivos. Esse método é apresentado como sem transfer learning; com classe negativa na Seção 5.

$\mathrm{Na}$ terceira abordagem, foi realizado o retreinamento apenas da última camada da rede, utilizando a técnica conhecida como transfer learning, com o objetivo de aproveitar o conhecimento retido no modelo pré-treinado e ainda acelerar os testes, pois dessa forma o custo computacional é reduzido substancialmente. Esse método é apresentado como transfer learning + classe negativa na Seção 5.

Por fim, além de transfer learning e inserção da classe negativa, também foi realizado um ajuste na base de treinamento, sobretudo nas marcações das máscaras e ajustes dos contornos faciais. Esse método é apresentado como transfer learning + classe negativa + máscaras reajustadas na Seção 5.

Para as quatro abordagens citadas, o objetivo era realizar a classificação do gênero, cuja análise do resultado é quantitativa e baseada na acurácia, bem como na comparação das taxas de falso positivo e falso negativo, com a obtenção da curva Receiver Operating Characteristics (ROC). A análise dos resultados da segmentação do contorno corporal é feita de forma qualitativa e visual.

\subsection{Métricas}

A avaliação quantitativa do método aplicado para classificação de gênero foi realizada através das medidas de acurácia. A acurácia mede a precisão geral do algoritmo, isto é, calcula o número de acertos dividido pelo total de amostras. Ela é aplicável a este estudo principalmente por conta da proporcionalidade entre os dados de cada classe aqui abordada.

Pela característica binária da classificação de gênero, cabe a aplicação da medida da área abaixo da curva ROC, que possibilita mensurar o desempenho do classificador avaliando a variação mútua entre a sensibilidade e a especificidade do modelo (Prati et al., 2008).

\section{RESULTADOS E DISCUSSÕES}

Todos os treinamentos desse trabalho foram realizados utilizando uma GPU Nvidia GTX 1070ti com 8GB de memória, processador Intel Core i5 8400 e 8GB de memória RAM.

A Figura 5 mostra alguns dos resultados obtidos para a segmentação de corpo inteiro das pessoas nas imagens. À esquerda, em azul escuro, estão os resultados obtidos com Mask R-CNN utilizando backbone ResNet50 e à direita, em azul claro, os resultados obtidos com ResNet101. É possível verificar que os contornos são obtidos quase que em sua totalidade com boa qualidade, principalmente após a utilização da rede mais profunda, exceto para o trecho da imagem entre os braços e o tronco, quando ambos estão pouco afastados, o que pode ser melhorado com pósprocessamento ou ainda com uma boa prática de posicionamento do indivíduo.

Em testes realizados utilizando imagens obtidas de ambientes com adversidades como sombras e fundo heterogêneo também foram obtidos resultados interessantes de segmentação, mais uma vez demonstrando a eficácia do algoritmo nessa aplicação com tais condições, conforme mostra a Figura 6.

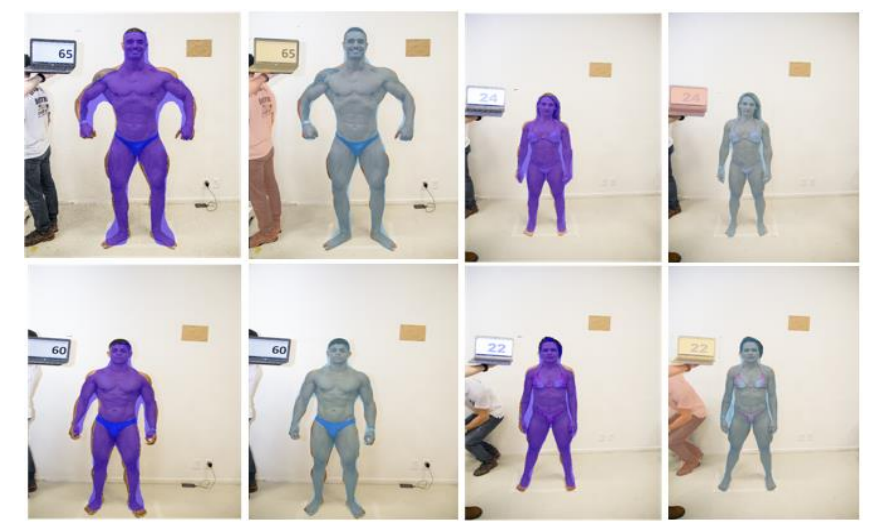

Fig. 5 Aplicação de Mask R-CNN com backbone ResNet50 (em azul escuro) e ResNet101 (em azul claro) 

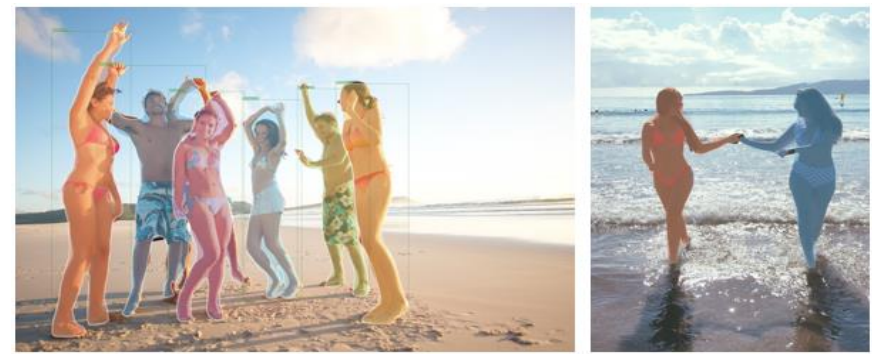

Fig. 6 Resultados da segmentação em fotos com sombras e fundo heterogêneo

Vale ressaltar que o algoritmo é robusto o suficiente para lidar com imagens contendo indivíduos em diferentes tamanhos e escalas, além de condições adversas de iluminação, inclusive com saturação e sombras, conforme pode ser observado na Figura 7.

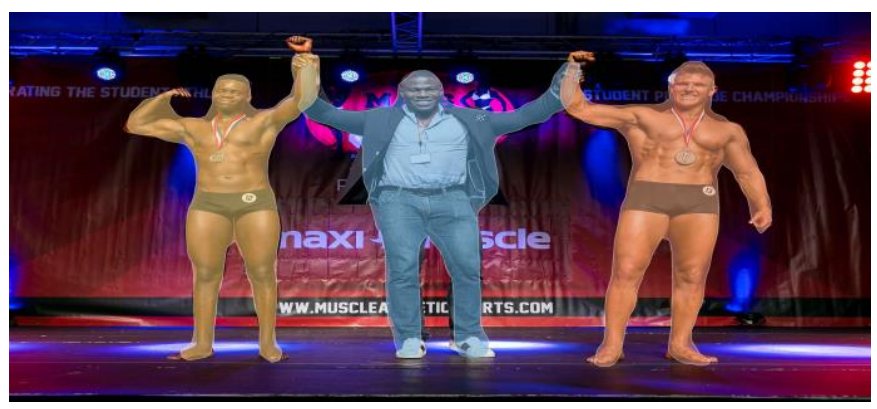

Fig. 7 Segmentação de múltiplas instâncias em ambiente não controlado

Finalmente, a Figura 8 mostra alguns resultados obtidos para a segmentação de faces e classificação de gênero, onde foi possível obter bons resultados de classificação mesmo quando o contorno não é perfeitamente encaixado na face. A classificação de gênero é correta mesmo em casos que a pessoa não está com a face perpendicular para a câmera, ou está fazendo algum movimento como um sorriso ou uma rotação da cabeça em relação à câmera. É importante citar que na base de treinamento foram utilizadas imagens com essas características. Apesar disso, no caso da obtenção da imagem para a aplicação do somatotipo a pessoa sempre estará com a face perpendicular à câmera.

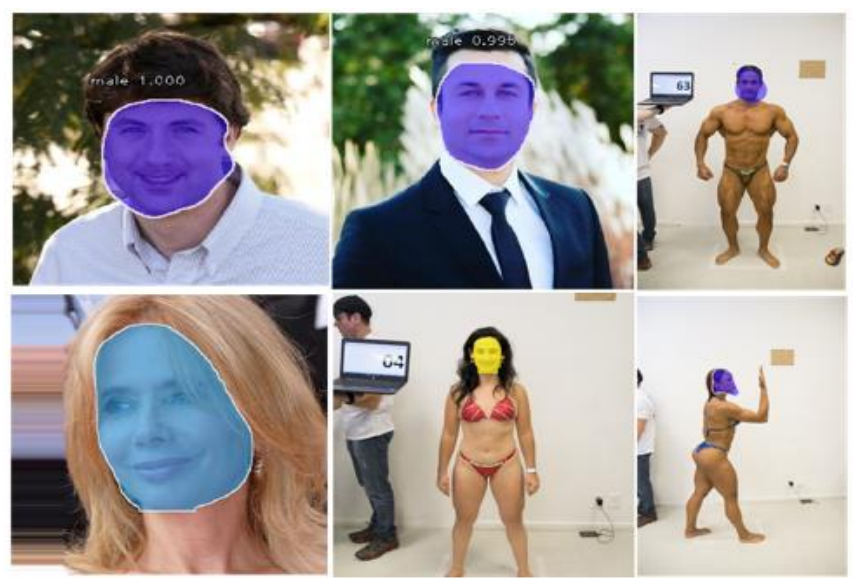

Fig. 8 Segmentação de face e classificação de gênero
A Tabela 1 mostra os resultados da acurácia de classificação.

Tabela 1. Acurácia da classificação de gênero

\begin{tabular}{lll}
\hline & \multicolumn{2}{c}{ Acurácia } \\
\hline & MASC & FEM \\
\hline $\begin{array}{l}\text { sem transfer learning; sem classe } \\
\text { negativa }\end{array}$ & $83,1 \%$ & $76,3 \%$ \\
\hline $\begin{array}{l}\text { sem transfer learning, com classe } \\
\text { negativa }\end{array}$ & $84,0 \%$ & $79,1 \%$ \\
\hline $\begin{array}{l}\text { transfer learning + classe negativa } \\
\text { transfer learning + classe negativa }+\end{array}$ & $\mathbf{9 5 , 4 \%}$ & $\mathbf{8 7 , 7 \%}$ \\
\hline \begin{tabular}{l} 
máscaras reajustadas \\
\hline
\end{tabular}
\end{tabular}

As abordagens realizadas sem transfer learning se mostraram menos eficazes, mesmo quando realizado treinamento com imagens da classe negativa, o que pode ser observado na primeira e segunda linha da Tabela 1. A inserção de imagens não rotuladas não resultou em melhoria significativa no resultado, apenas contribuiu com um aumento de aproximadamente $3 \%$ da acurácia para o gênero feminino.

A utilização do transfer learning isoladamente também não resultou em um aumento significativo da acurácia, a princípio. Por outro lado, a grande vantagem da terceira abordagem foi a diminuição do custo computacional, uma vez que todo o treinamento foi realizado com dez mil iterações e trinta épocas e a redução do tempo de treinamento foi de cerca de $50 \%$ do tempo demandado sem a utilização da técnica, resultando em aproximadamente quatro horas de treinamento. A quarta abordagem de treinamento trouxe a melhoria mais perceptível nos resultados, após ser realizado um reajuste na base de treinamento, que consistiu no refinamento das bordas das máscaras, ajustando os contornos das faces e as coordenadas dos bounding boxes delas.

Com o modelo final treinado, além da métrica de acurácia, já citada, foi obtida a curva ROC para avaliação da qualidade do classificador, onde foi obtido um valor de 0,719 para a área sob a curva, o que demonstra que o método resultou em um bom classificador, porém ainda há um caminho a ser explorado para torná-lo excelente. Há a expectativa de que esse resultado possa ser melhorado utilizando imagens com resolução mais alta nos treinamentos e testes, bem como a aplicação de técnicas de data augmentation.

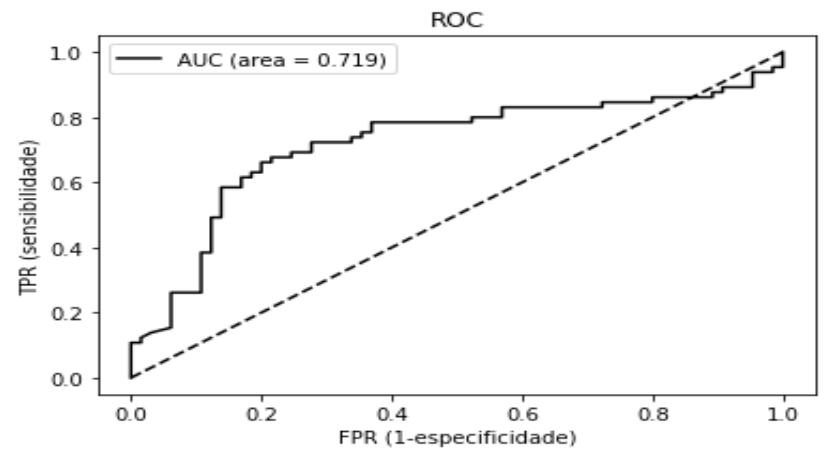

Fig. 8 Curva ROC obtida para o classificador de gênero 


\section{CONCLUSÕES}

O objetivo desse trabalho foi aplicar a técnica Mask R-CNN utilizando backbones ResNet na tarefa de segmentação de pessoas e classificação de gênero analisando faces em imagens de ambientes não controlados, de forma a possibilitar a construção de um algoritmo que forneça dados essenciais para serem integrados a um sistema de avaliação automática de somatotipo.

Foi possível observar que a técnica testada é robusta e consegue realizar com boa qualidade a tarefa de segmentação de pessoas em ambientes não controlados, podendo servir de base para um sistema de baixo custo de classificação automática de somatotipo, contribuindo, portanto, para tal pesquisa em desenvolvimento. Além disso, foi concluído que foi possível alcançar um classificador com nível de acurácia que viabiliza a tarefa da obtenção do gênero com base em análise facial.

Como trabalhos futuros, propõe-se aplicar diferentes arquiteturas de redes profundas para a classificação de gênero e compará-las com a ResNet50. Depois, utilizar o resultado da segmentação dos contornos corporais como entrada de um sistema de estimativa de somatotipo, visando avaliar de forma quantitativa o resultado dessa segmentação no algoritmo de estimativa de somatotipo desenvolvido pelo trabalho de referência para essa pesquisa, feito por Gonçalves (2017).

\section{AGRADECIMENTOS}

Os autores agradecem o apoio financeiro à pesquisa do projeto da Fundação de Amparo à Pesquisa do Espírito Santo (FAPES), número 598/2018. O trabalho de E. O. T. Salles foi apoiado pela FAPES, sob a bolsa 244/2016.

\section{REFERÊNCIAS}

Deng, L., (2014). A tutorial survey of architectures, algorithms, and applications for deep learning. APSIPA Transactions on Signal and Information Processing, v. 3, pp. 1-29

Da Silva, P. R. P.; Trindade, R.; De Rose, E. H., (2003). Composição corporal, somatotipo e proporcionalidade de culturistas de elite do Brasil. Rev Bras Med Esporte v. 9, n. 6 , pp. 403-407

Fukushima, K. (1988). Neocognitron: A hierarchical neural network capable of visual pattern recognition. Neural networks 1.2 (1988): pp. 119-130

Girshick, R., (2014). R-CNN - Rich feature hierarchies for accurate object detection and semantic segmentation. The IEEE Conference on Computer Vision and Pattern Recognition (CVPR), June 2014.

Girshick, R. (2015). Fast R-CNN. ICCV '15 Proceedings of the 2015 IEEE International Conference on Computer Vision (ICCV), pp. 1440-1448.

Gonçalves, T. O.; Ciarelli, P. M.; Coco, K. F., (2016). Uma análise da viabilidade de estimar somatotipos de fisiculturistas através de medições em imagens. XXI Congresso Brasileiro de Automática - CBA2016, pp. 3416-3421.
Gonçalves, T. O., (2017). Identificação do Somatotipo de Fisiculturistas Através de Imagens Digitais. Dissertação de Mestrado, Programa de Pós-Graduação em Engenharia Elétrica da Universidade Federal do Espírito Santo, Vitória, Espírito Santo, Brasil.

He, K.; Zhang, X.; Ren, S.; Sun, J., (2016). Deep Residual Learning for Image Recognition. Conference on Computer Vision and Pattern Recognition (CVPR). June 2016.

He, K.; Gkioxari, G.; Dollár, P.; Girshick, R., (2017). Mask R-CNN. Conference on Computer Vision and Pattern Recognition (CVPR). June 2017

Jaksic, D.; Cvetković, M., (2009). Neural network analysis of somatotype differences among males related to the manifestation of motor abilities, Acta Kinesiologica 3(1): 107-113

Krizhevsky, A.; Sutskever, I.; Hinton, G. E., (2012). ImageNet Classification with Deep Convolutional Neural Networks. Communications of the ACM, 60(6): 84-90.

LeCun, Y.; Boser, B.; Denker, J.; Henderson, D.; Howard, R.; Hubbard, W.; Jackel, L. (1989). Backpropagation applied to handwritten zip code recognition. Neural Computation 1(4): 541-551.

Li, Y,; Qi, H.; Dai, J.; Ji, X.; Wei, Y., (2017). Fully convolutional instance-aware semantic segmentation. Conference on Computer Vision and Pattern Recognition (CVPR), July 2017.

Long, J.; Shelhamer, E.; Darrell, T., (2015). Fully convolutional networks for semantic segmentation, Conference on Computer Vision and Pattern Recognition (CVPR), July 2015.

Parolin, A., (2011). Segmentação de imagens de pessoas em tempo real para videoconferências. Dissertação de Mestrado, Universidade do Vale do Rio dos Sinos, UNISINOS, Brasil.

R. C. Prati, G. E. A. P. A. Batista e M. C. Monard, (2008). Curvas ROC para avaliação de classificadores. IEEE Latin America Transactions[S.l.], 6(2): 215-222

Ren, S.; He, K.; Girshick, R.; Sun, J., (2015). Faster R-CNN: Towards real-time object detection with region proposal networks, IEEE Transactions on Pattern Analysis \& Machine Intelligence, 39(6): 1137-1149.

Rothe, R.; Timofte, R.; Gool, L.V., (2016). Deep expectation of real and apparent age from a single image without facial landmarks, International Journal of Computer Vision, v. 126, pp. 144-157.

Russel, B.; Torralba, A.; Murphy, K.; Freeman, W. T.; (2008). LabelMe: a database and web-based tool for image annotation, International Journal of Computer Vision, v. 77, p. 157-173

Saxena, A.; Chung, S. H.;Ng, A. Y., (2005). Learning Depth from Single Monocular Images, NIPS'05 Proceedings of the 18th International Conference on Neural Information Processing Systems, pp. 1161-1168

Sheldon, W. H., Stevens, S. S. e Tucker, W. B. (1940). The varieties of human physique. Oxford, England: Harper.

Viola, P. e Jones, M. J. (2004). Robust real-time face detection, International Journal of Computer Vision 2(57): 137-154. 